\title{
Printing and Curing of Conductive Ink Track on Fabric using Syringe Deposition System with DLP Projector and Hot Plate
}

\author{
Rd. Khairilhijra Khirotdin ${ }^{1}$, Mohamad Izwan Saifudin Mohamad Zainuri ${ }^{2}$, Afiqah Izzati \\ Ishak ${ }^{3}$, Nurhafizzah Hassan ${ }^{4}$, Khairu Kamarudin ${ }^{5}$, Mohd. Rasidi Ibrahim ${ }^{6}$, Reazul Haq \\ Abdul Haq ${ }^{7}$, Mustafa Ibrahim ${ }^{8}$, Nasuha Sau'de ${ }^{9}$, Omar Mohd. Faizan Marwah ${ }^{10}$ \\ 1,2,5,6,7,8,9,10 Faculty of Mechanical and Manufacturing Engineering, Universiti Tun Hussein Onn \\ Malaysia, 86400 Parit Raja, Batu Pahat, Johor, Malaysia \\ ${ }^{3,4}$ Faculty of Engineering Technology, Universiti Tun Hussein Onn Malaysia, \\ 86400 Parit Raja, Batu Pahat, Johor, Malaysia
}

\begin{abstract}
Printing is a technique to transfer ink onto substrates to create pattern and syringe deposition system has shown some great potential in printing due to its ability to produce filamentary bead tracks which is important concerning conductivity and easily adopted on conformal surfaces which could not be realized by conventional technique. Fabrics with integrated electrical features able to create intelligent articles and may potentially open up new perspective areas of application in textile printing. However, the applicability of this technique on fabrics remains unknown which the ink used has to meet certain requirements including high electrical conductivity, resistance to oxidation, dry out without clogging, good adhesion with suitable viscosity and surface tension. Thus, there is a need to do this study which is to determine the feasibility of syringe deposition system to print a conductive ink tracks using silver epoxy-based conductive ink on fabric substrate via lycra material. This study is also aim to investigate the feasibility of using DLP projector with hot plate as another source of heat to be used in curing the ink tracks on fabric. The effect of printing and curing parameters to the characteristics and conductivity of the ink track is investigated. Several mechanical and electrical tests were also administered to determine the cure, hardness, adhesion and resistance level of the ink tracks. The results obtained were as expected which higher printing speed and lower deposition height used, a narrower and thinner ink tracks were produced. Sample with $4 \mathrm{~mm} / \mathrm{s}$ of printing speed and deposition height of 1 $\mathrm{mm}$ resulted in dimension closer to the targeted dimension. The longer curing time and higher temperature used, a lower resistance is produced. The lowest resistance achieved is $0.9 \Omega$ cured at $150^{\circ} \mathrm{C}$ for 60 minutes. The conductivity of the ink track was affected by curing process and crosssectional area of the ink track. It is proven that a syringe deposition system is capable of printing the ink track and DLP projector with hot plate is suitable to cure the ink track properly on fabric.
\end{abstract}

*Corresponding author: khairil@uthm.edu.my 


\section{Introduction}

Printing is a method used to transfer metallic conductive ink into a substrate to create electronics structure. Among all printing methods, syringe deposition system is one of the method to apply liquid solution with precision and high speed and capable of coping with variety of materials such as conductive ink and it has shown some great potentials in printing conductive ink due to its ability to produce filamentary bead tracks which is important with respect to conductivity [1]. Another prospect of this technology is it could be adopted especially on non-planar and curvature surfaces where conventional printing technique could not be realized [2]. However, the applicability of this technique on fabrics remains unknown which the conductive ink used has to meet certain requirements including high electrical conductivity, resistance to oxidation, dry out without clogging, good adhesion with suitable viscosity and surface tension. Therefore, there is a need to do this study in which it will focus on finding the right materials and technique to be used for printing electronics structures on fabrics.

In the mean time, conductive ink has been widely utilized in various applications of electronic components [3]. Since silver has high stability against air and one of the best conductivity, silver conductive ink is widely used to manufacture conductive patterns in electronic devices [4]. Conductive ink is mundanely come in liquid form and requires curing to expose its metallic contents. Curing is a process to crosslink the polymer materials and it is usually done after the conductive ink has been printed on substrates. The curing process is essential to eliminate the unwanted solvents and evaporate the binder in the substrate. In this study, it is focused on how to cure the conductive ink on fabric material. There are several type of curing process available using several technologies including oven, laser, ultraviolet (UV) radiation and electron beam. Some of these technologies suffer from drawbacks such as oven curing process is done in a closed system and the heat generated is presumably double (flash cure) from its designated temperature set if it is not properly controlled while laser is considered expensive system and requires a proper control especially on its power which could damage the substrate. Thus, a more suitable and simpler curing method is needed whereby a concept of gradually curing process is possible to properly cure the ink track without damaging the substrate. A Direct Light Projector (DLP) was seen capable of polymerizing photopolymer materials [5]. In addition, hot plate as well is promising since it was used previously to cure several conductive inks on different substrates [4]. Thus, by combining them might properly cure both areas of the inks since DLP projector is focused on curing the top part of the inks while hot plate cured the bottom part of the ink. This helps the heat to evenly distribute and a higher conductivity of the ink tracks could be realized without damaging the substrate. In addition, it could also decrease the cost as the projector and hot plate is quite cheap, easy to set up and portable. An investigation is performed to determine the optimum curing time and temperature to cure the ink track from syringe deposition system on fabric. Fabrics materials with integrated electrical features able to create intelligent articles with wide ranging of applications such as sports, work wear, health care, safety and others. These advances in technology may open new perspective for such areas of application in textile printing as sampling, strike-offs and customization.

\section{Objective}

The objective of this study is to determine the feasibility of syringe deposition systems to print a conductive ink tracks using silver epoxy -based conductive ink on fabric substrate via lycra material. This study is also aim to investigate the feasibility of using DLP projector with hot plate as another source of heat to be used in curing the ink tracks on fabric. The results obtained are used to determine the relationship between printing and curing 
parameters to the morphological, mechanical and electrical properties of the electronics structure printed.

\section{Methodology}

\subsection{Pattern design}

The pattern design to be printed onto the substrate is a strain gauge pattern. Flexibility and various sizes are the advantages for strain gauge as a chosen electronic structure for the study. The total length of the strain gauge is $219 \mathrm{~mm}$ with a $2 \mathrm{~mm}$ of ink track width as depicted in Figure 1. Taguchi's method was used to identify the number of experiments needed in order to save time and cost.

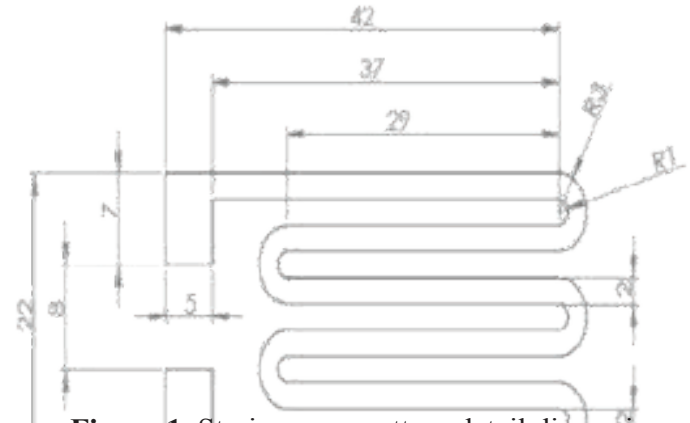

Figure 1. Strain gauge pattern detail dimension (unit in $\mathrm{mm}$ )

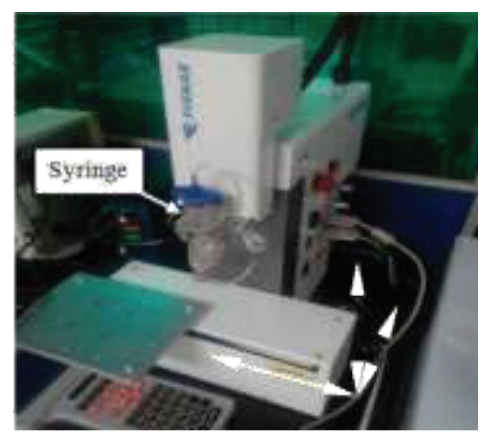

Figure 2. Syringe deposition system

\subsection{Printing and curing process}

Syringe deposition system (Model: FISNAR F4200N.1) was used as a deposition method in the study as shown in Figure 2. It is non -contact types of printing technique and using the syringe deposition system gave a slight advantage since it allows printing on conformal surfaces while most of printing technologies could not. Printing with syringe deposition system mostly resulted a semi- circular shape ink track in contrast with other printing technologies especially ink jet printing where a rectangular shape is always printed [6] as illustrated in Figure 3. Printing parameters including the nozzle diameter, pressure, printing speed and height deposition of the nozzle from the substrate is the influence factor to the physical characteristics of the ink droplet. Viscosity of the ink also played a major role and the printing parameters selected in this study are tabulated in Table 1 while the other parameters are set to be constant. 


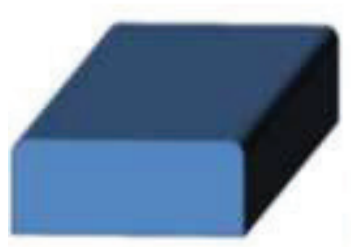

Rectangular

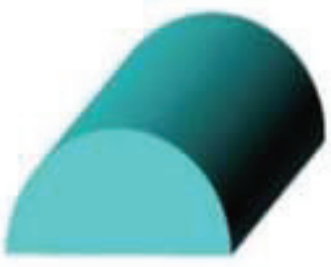

Semicircular are

Figure 3. The ink droplet shape from inkjet and syringe deposition system [6].

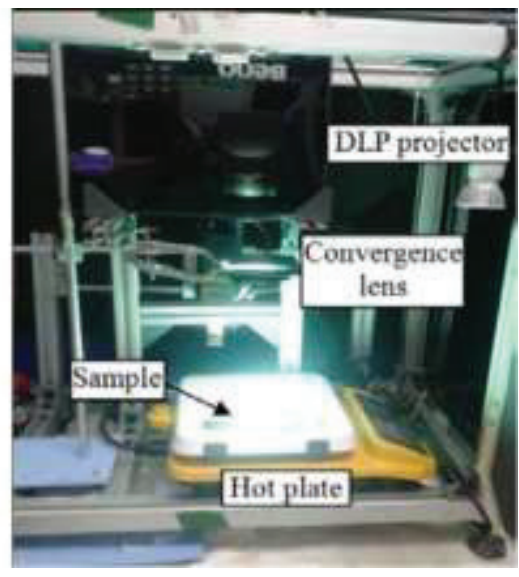

Figure 4. Experimental set up for curing process.

On the other hand, the curing process is a process which both chemical reaction and physical action takes place to make the ink become harder, tougher or more stable linkage. Since the conductive ink comes in liquid form and required curing to expose its metallic contents, the unwanted material is needed to be removed so that the conductive ink track could obtain better electrical properties. A combination of DLP and hot plate was used for curing process and the experimental set up is depicted in Figure 4 where DLP is set to focus on curing the top part while hot plate is set to cure the bottom part of the ink. Thus, by combining them might properly cure both areas of the inks (top and bottom) since the heat may evenly distributed and a higher conductivity of the ink tracks could be realized without damaging the substrate. The curing parameters selected including curing time and temperature are tabulated in Table 1 while the other parameters are set to be constant.

Table 1. Printing and curing parameters selected for the experiment.

\begin{tabular}{|c|c|c|c|c|}
\hline No. sample & Printing Speed $(\mathrm{mm} / \mathrm{s})$ & Height deposition $(\mathrm{mm})$ & Curing time $(\mathrm{min})$ & Curing temperature $\left({ }^{\mathrm{O}} \mathrm{C}\right)$ \\
\hline 1 & 3 & 0.5 & 15 & 110 \\
\hline 2 & 4 & 1.0 & 30 & 120 \\
\hline 3 & 5 & 1.5 & 45 & 130 \\
\hline 4 & 6 & 2.0 & 60 & 140 \\
\hline 5 & 7 & 2.5 & 75 & 150 \\
\hline
\end{tabular}

\subsection{Material}

Viscosity is one of the most important properties when printing ink. Most conductive inks have surface tension and viscosity value which allow the droplet formation to be dispensed. This is due to the conductive ink mostly using water or polymer as the solvent in controlling viscosity of the ink. Viscosity of the ink is dependent on temperature and it is important to recognize that a large fluctuation in temperature can change viscosity and ultimately the ink performance. Silver epoxy-based ink (Model: AG 806) as shown in Figure 5 is the conductive ink used for the study and it is chosen since it has higher anti oxidation properties 
rather that copper based ink. A direct usage of the ink used is not possible due to high solid fraction thus an adjustment of viscosity via dilution with toluene solvent is necessary. A vibro-viscometer was used to measure the viscosity and the viscosity is required to be reduced to one sixth of the original viscosity to ensure the ink could be dispensed without clogging. The dilution process is performed by adding $0.5 \mathrm{ml}$ of toluene for ten times as tabulated in Table 2 without altering too much its solid content.

Table 2. Result of the viscosity reductive ink with toluene.

\begin{tabular}{|c|c|c|} 
Trial & $\begin{array}{c}\text { Volume of } \\
\text { toluene }(\mathrm{ml})\end{array}$ & Viscosity (mPa.s) \\
\hline 1 & 0 & 7880 \\
\hline 2 & 0.5 & 6054 \\
\hline 3 & 0.5 & 4527 \\
\hline 4 & 0.5 & 3764 \\
\hline 5 & 0.5 & 3000 \\
\hline 6 & 0.5 & 2500 \\
\hline 7 & 0.5 & 2365 \\
\hline 8 & 0.5 & 2230 \\
\hline 9 & 0.5 & 1730 \\
\hline 10 & 0.5 & 1580 \\
\hline 11 & 0.5 & 1230 \\
\hline
\end{tabular}

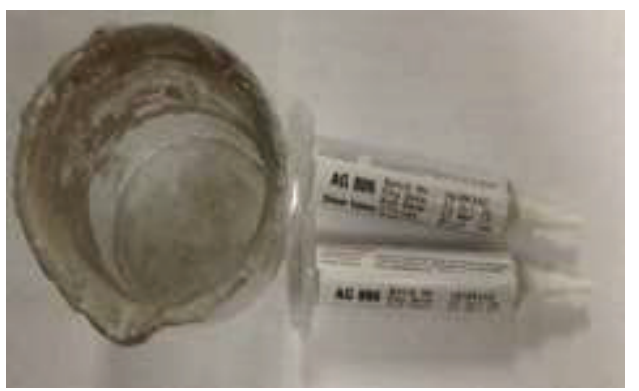

Figure 5. Silver epoxy-based ink (AG 806) Produced by Penchem Technologies.

\subsection{Substrate}

Lycra (spandex) as shown in Figure 6 (a) was used as a substrate material for the experiment and it is commonly used in sportswear due to it is light, smooth and soft. Lycra was chosen since the aim is to embed sensor on cloth and lycra materials is much suitable because it is a type of compression wear (highly stretching up to $500 \%$ of their original length) where under compression it clings strict to the skin and quite literally squeezing the muscles below without irritation hence helps to increase the sensor sensing capabilities. In addition, to have a good substrate in fabrics, all requirements must be fulfilled such as the smoothness of the 
substrate, the melting point of the substrate, the compressibility and the ink receptivity [7]. Rubber dye ink is coated on lycra surface (refer Figure 6 (b)) prior to printing process to prevent the fabric from absorbing the ink. The coating as well act as an insulator to cover any gaps presence in fabric structure (refer Figure 6 (a)) so that the ink would not dissipate much to the environment.

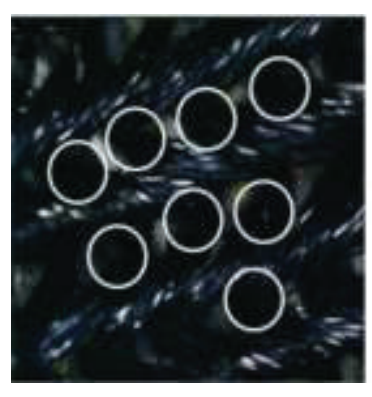

(a)

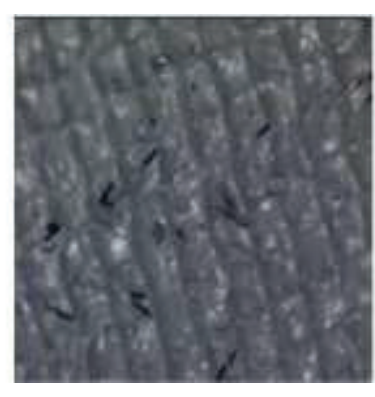

(b)

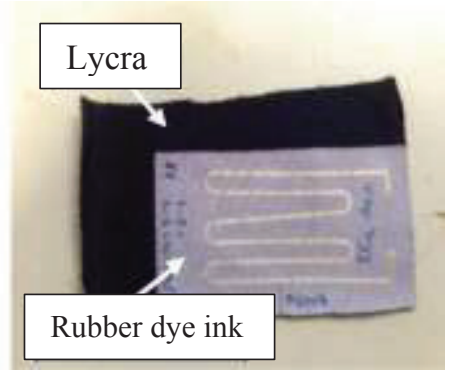

(c)

Figure 6. Micro-structure of lycra's surface before and after coating (a) gaps present (denoted as white circle) (b) gaps covered with rubber dye ink (c) lycra covered with rubber dye ink

\subsection{Mechanical and electrical test}

The electrical tests were conducted to obtain the resistance by measuring the resistance using digital millimeter. The mechanical tests were also performed including the manual scratch test, adhesion and hardness test. The manual scratch test is to determine the cure level of the ink track while the adhesion test via the tape test method is to assess the adhesion level of the ink track. HMV micro-hardness test is employed to determine the hardness level of the ink track.

\section{Result and discussion}

\subsection{Printing process}

All fabric substrates were first screen printed with rubber dye ink before the experiments begin. This is done to avoid the silver conductive ink from leaking into the fabric. The optimum parameters were selected from the preliminary experiments performed earlier and the other parameters were set to be constant including the viscosity of the conductive ink $(1,230 \mathrm{mPa} . \mathrm{s})$, the nozzle tip size $(0.58 \mathrm{~mm})$ and the pressure applied ( 0.8 bar). Table 3 and 4 below shows the result for experimental of printing using silver conductive ink. 
Table 3. Result of the effect of variation of printing speed to morphology of the ink track at height deposition of $1 \mathrm{~mm}$

\begin{tabular}{|c|c|c|c|c|c|c|c|c|}
\hline Sample & $\begin{array}{c}\text { Deposition } \\
\begin{array}{c}\text { Height } \\
(\mathrm{mm})\end{array} \\
\end{array}$ & $\begin{array}{l}\text { Printing } \\
\text { Speed } \\
(\mathrm{mm} / \mathrm{s}) \\
\end{array}$ & $\begin{array}{l}\text { Width } \\
(\mathrm{mm})\end{array}$ & $\begin{array}{l}\text { Designated } \\
\text { width }(\mathrm{mm})\end{array}$ & $\begin{array}{l}\text { Thickness } \\
(\mathrm{mm})\end{array}$ & $\begin{array}{c}\text { Length } \\
(\mathrm{mm})\end{array}$ & $\begin{array}{l}\text { Area } \\
\left(\mathrm{mm}^{2}\right)\end{array}$ & $\mathrm{A} / \mathrm{L}(\mathrm{mm})$ \\
\hline 1 & \multirow{5}{*}{1} & 3 & 2.258 & \multirow{5}{*}{2} & 1.207 & \multirow{5}{*}{42} & 2.725 & 0.0648 \\
\hline 2 & & 4 & 1.965 & & 0.231 & & 0.454 & 0.0108 \\
\hline 3 & & 5 & 1.850 & & 0.218 & & 0.403 & 0.0096 \\
\hline 4 & & 6 & 1.425 & & 1.125 & & 1.603 & 0.0382 \\
\hline 5 & & 7 & 1.080 & & 0.790 & & 0.853 & 0.0203 \\
\hline
\end{tabular}

Table 4. Result of the effect of variation of deposition height to morphology of ink track at printing speed of $4 \mathrm{~mm} / \mathrm{s}$

\begin{tabular}{|c|c|c|c|c|c|c|c|c|}
\hline Sample & $\begin{array}{l}\text { Printing } \\
\\
\begin{array}{l}\text { Speed } \\
(\mathrm{mm} / \mathrm{s})\end{array} \\
\end{array}$ & $\begin{array}{c}\text { Deposition } \\
\begin{array}{c}\text { Height } \\
(\mathrm{mm})\end{array}\end{array}$ & Width (mm) & $\begin{array}{l}\text { Designated } \\
\text { Width }(\mathrm{mm})\end{array}$ & $\begin{array}{l}\text { Thickness } \\
\text { (mm) }\end{array}$ & $\begin{array}{c}\text { Length } \\
(\mathrm{mm})\end{array}$ & $\begin{array}{c}\text { Area } \\
\left(\mathrm{mm}^{2}\right)\end{array}$ & $\mathrm{A} / \mathrm{L}(\mathrm{mm})$ \\
\hline 1 & \multirow{5}{*}{4} & 0.5 & 1.885 & \multirow{5}{*}{2} & 0.632 & \multirow{5}{*}{42} & 1.191 & 0.0284 \\
\hline 2 & & 1.0 & 1.965 & & 0.711 & & 1.397 & 0.0333 \\
\hline 3 & & 1.5 & 1.530 & & 0.654 & & 1.001 & 0.0238 \\
\hline 4 & & 2.0 & 1.618 & & 0.944 & & 1.527 & 0.0364 \\
\hline 5 & & 2.5 & 1.505 & & 0.627 & & 0.944 & 0.0225 \\
\hline
\end{tabular}

All results were plotted as shown in Figure 7 (a) which it clearly seen that the line width and line thickness of ink track were affected by the variation of printing speed. When the printing speed is faster, the width of the ink tracks were smaller and far from the expected width size. This is due to the time for the ink to be dispensed is shorter and at the same time the thickness also thinner. The changes in thickness were not too obvious compared with the width of the ink track. In addition, the deposition height also gives an impact to the line width and line thickness. If the deposition height used is higher, the ink droplet is not stable result in a wrinkle condition of track and in some condition incontinuous ink tracks were produced. Thus, the distance between the nozzle tip and the substrate is needed to be kept close to ensure a continuous and a straight ink track is produced. Figure 7 (b) shows the changes in width and thickness of the ink track with variation of deposition height at constant printing speed of $4 \mathrm{~mm} / \mathrm{s}$. It was observed that the width of the track changed to a narrower ink track whereas not much changed occurred for the thickness of the ink track. 


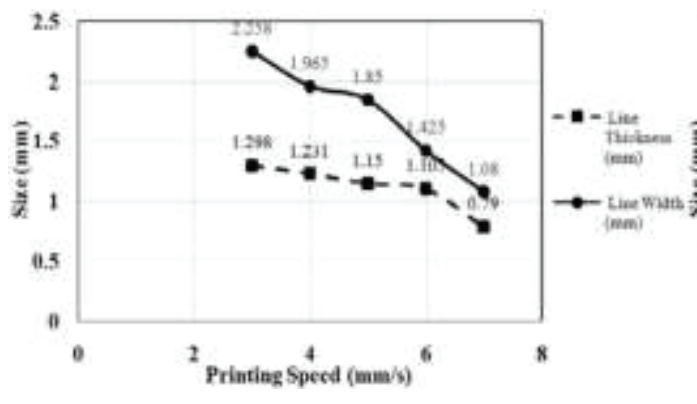

(a)

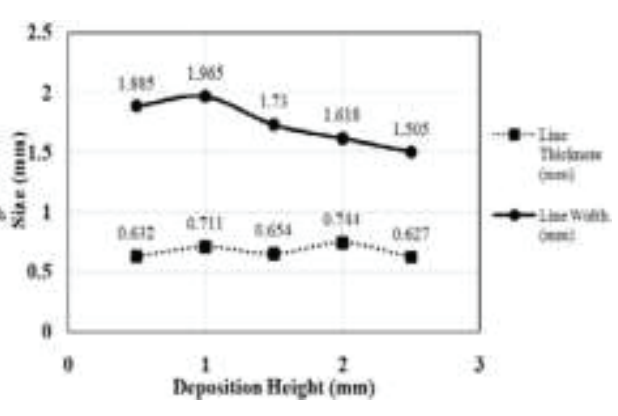

(b)

Figure 7. Changes of width and thickness of the ink track with (a) variation of printing speed at deposition height of $1 \mathrm{~mm}$ (b) variation of deposition height at printing speed of $4 \mathrm{~mm} / \mathrm{s}$.

\subsection{Curing Process}

Figure 8 (a) shows the resultant changes in resistance obtained against variation of time and temperature selected. It was observed that the increase in temperature resulted in decrease in resistance. The trend also shows the resistance also decreases as the curing time increased. Furthermore, at temperature of $120^{\circ} \mathrm{C}$ shows a constant decreased in resistance for five different time intervals. However, there are some anomalies happened at temperature $110^{\circ} \mathrm{C}$ and $130^{\circ} \mathrm{C}$ where the resistance suddenly becomes higher at curing time of 45 minutes due to the temperatures were not properly controlled. At temperature of $140^{\circ} \mathrm{C}$ and $150^{\circ} \mathrm{C}$, the trend shows decreasing in resistance for all curing time except at 75 times due to over cure phenomenon. The lowest resistance achieved is $0.9 \Omega$ cured at $150^{\circ} \mathrm{C}$ for 60 minutes. Besides, Figure 8 (b) shows the changes in conductivity against variation of curing time and temperature selected where the conductivity was found by calculation using equation (1) where the conductivity, $\sigma$ of the ink track is based on the size of cross-sectional area, $A$ of the ink track and the resistivity, $\rho$ as shown in the following equation (1) and (2).

$$
\begin{aligned}
& A=\text { Thickness } x \text { Width } \\
& \text { Conductivity, } \sigma=\frac{1}{\rho}
\end{aligned}
$$

It shows that the conductivity increased as the curing time increased. At 60 minutes of curing time, the conductivity reached its peak for both curing temperatures at $140^{\circ} \mathrm{C}$ and $150^{\circ} \mathrm{C}$. However, at curing time of 75 minutes, both lines had sudden dropped due to over cured phenomenon where some silver metals were burnt out and this decrease the conductivity. 


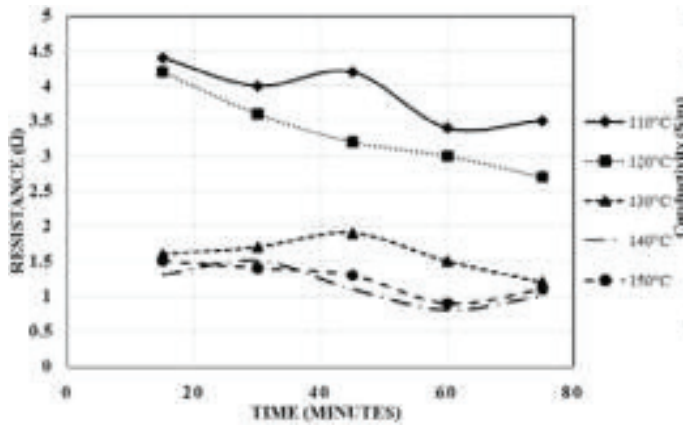

(a)

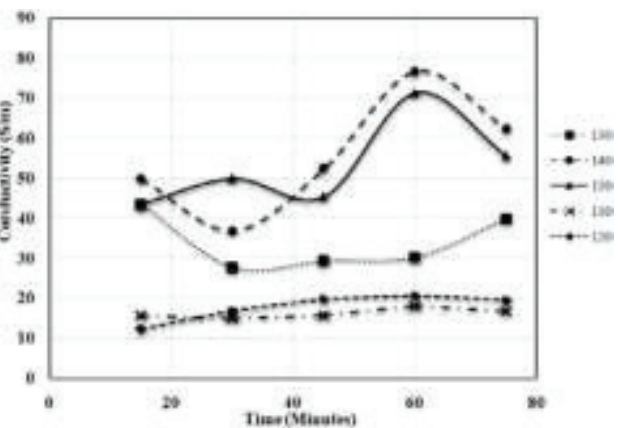

(b)

Figure 8. (a) Changes of resistance for variation of curing time at several curing temperatures (b) Changes of conductivity for variation of curing time at several curing temperatures.

\subsection{Morphology analysis}

The strain gauge ink track structure was analysed using optical microscope to determine its physical properties including its track width, thickness and cross-sectional shape after cured. The cross-sectional shape should form a semi-circular like shape and Figure 9 (a) shows a shape of semi-circular shape of the ink track structure as mentioned by [7]. The results of the physical properties of the ink track have been tabulated earlier in Table 3 and 4 . The following equation (3) has been used to calculate the percentage of dimensional accuracy of the ink track dimension to its designated dimension. It is performed to determine the effectiveness of the syringe deposition system in printing conductive ink on lycra substrate. The results for the dimensional accuracy measurement are shown in Figure 10 (a) and (b). It was observed that the printing process by syringe deposition system produced a dimension closed enough to the designated design size due to the suitable parameter used. It was observed that the optimum parameter selected (deposition height of $1 \mathrm{~mm}$ and printing speed of $4 \mathrm{~mm} / \mathrm{s}$ ) is verified through the percentage of accuracy acquired since the selected parameters manage to achieve the physical characteristic of the designated ink track's size.

$$
\text { Dimensional accuracy } \%=\frac{\text { sample result }}{\text { expected result }} \times 100 \%
$$

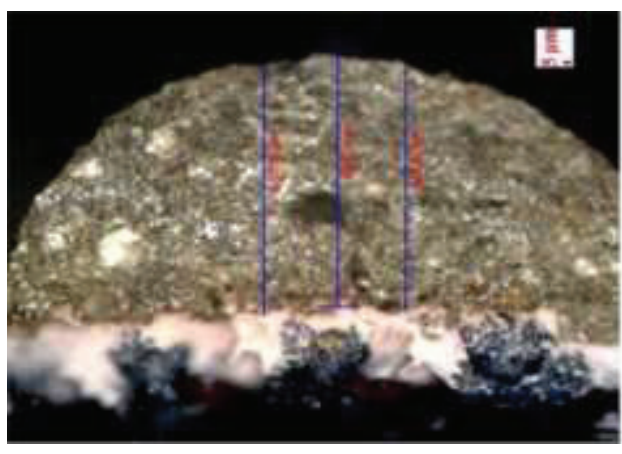

(a)

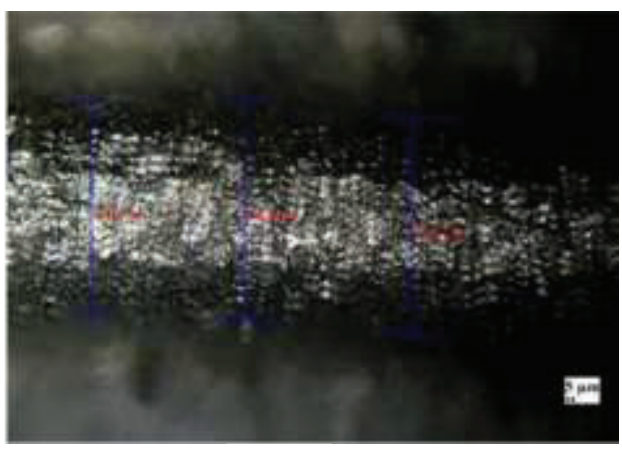

(b)

Figure 9. (a) Cross-section view of the ink track showing the thickness (b) A view of the width of the track showing a fine track edges. 


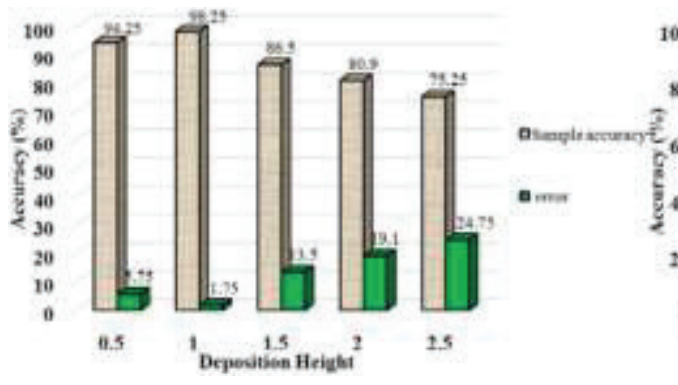

(a)

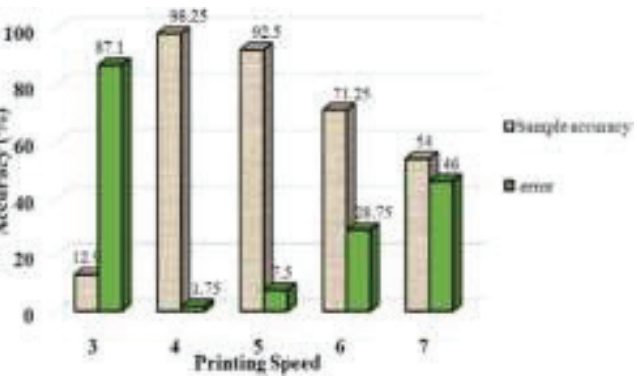

(b)

Figure 10. Percentage of dimensional accuracy of the width with (a) variation of deposition height

(b) variation of printing speed

\subsection{Mechanical Test}

Mechanical test is important to identify whether the ink track has been fully cured, its hardness and adhesion level after cured. The manual scratch test is performed to detect the smear from the ink track using a needle right after the sample was cured. The hardness test is conducted where the diamond indenter with certain load is applied onto the ink track resulted in a diamond shape. Lastly, the adhesion test is performed using the tape test method in which the $3 \mathrm{M}$ tape is applied on the ink track and smoothed by the eraser. The tape is pulled off rapidly at an angle of $180^{\circ}$ and the adhesion level is assessed from the range $0 \mathrm{~B}$ to 5B as stated by ASTM D3359. Based on the result from Figure 11, the graph shows a few fluctuated points at the beginning; however the overall trend shows a clear trend of increasing adhesion level as the curing time and temperature increased. Similar trend was observed on the results of hardness level as depicted in Figure 12 which the hardness level of the ink tracks showed inclination trend similar to the previous adhesion test. It was found that when the cured temperature and the time increased, the hardness level of the ink tracks was also increased accordingly.

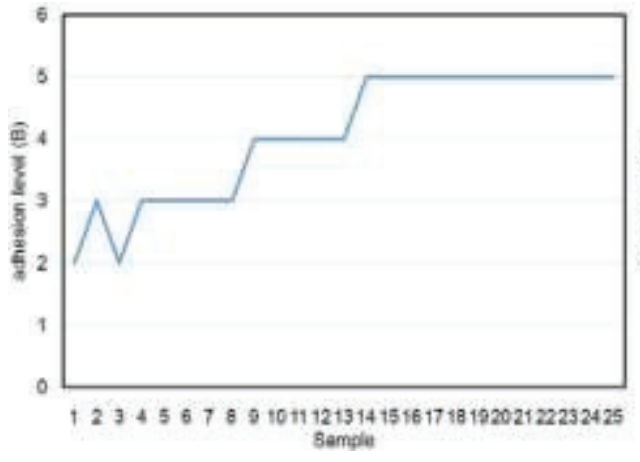

Figure 11. Changes of adhesion level for all samples. samples.

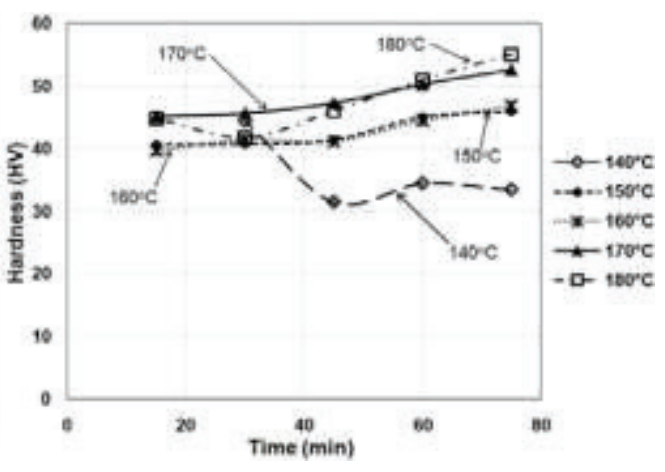

Figure 12. Changes of hardness level for all 


\section{Conclusion}

The objective of the study which is to investigate the feasibility of printing and curing the silver conductive ink tracks on fabric using syringe deposition technique and a combination of DLP projector and hot plate is achieved. The strain gauge pattern was successfully printed and properly cured on lycra substrate. The printing parameters involved which is the deposition height and the printing speeds were carefully controlled to achieve the desired ink track's size. The accuracy of the ink track dimension and cross-sectional shape of the ink track are depended on the printing parameters selected. The preliminary experiments are required to prevent any problem that may occur in the actual experiments. It also intended to save cost and time during the actual experiment. Besides, DLP projector with hot plate has proven capable of properly cure the silver conductive ink by means of evaporating the binders and unwanted solvent thus harden and cross linked the metallic particles with the substrate. The resistance is much depending on curing time and temperature in which the higher the temperature and the longer the cure time used, the lower the value of resistance. On the other hand, conductivity is inversely proportional to the resistance in which when the curing time and temperature increases, the conductivity obtained also increases.

\section{Acknowledgement}

The authors gratefully acknowledge the support to the Ministry of Higher Education of Malaysia and University of Tun Hussein Onn Malaysia under the Research Acculturation Grant Scheme awarded (RAGS-R052) and giving the opportunity to attend and present at this conference.

\section{References}

1. E. Fearon, T. Sato, D.Wellburn, Thermal Effects of Substrate Materials Used in the Laser Curing of Particulate Silver Inks, Proc LANE Laser Assisted Net Shape Engineering,c379-390 (2007).

2. R. Khirotdin, M. F. Ngadiron, M. A. Mahadzir, N. Hassan, Performance Evaluation of Strain Gauge Printed Using Automatic Fluid Dispensing System on Conformal Substrates, $3^{\text {rd }}$. International Research and Innovation Summit (2017), Malacca, Malaysia.

3. K. Khirotdin, M. A. Nasir, K. Kamarudin, M. Ibrahim, Investigation of DLP Projector as an Energy Light Source to Cure Silver Conductive Ink, Applied Mechanics \& Materials, Vol. 773-774, p 13-19 (2015).

4. I. Kazani, C. Hertleer, G. De Mey, A. Schwarz, G. Guxho, L. V. Langenhove, Electrical conductive textiles obtained by screen printing. Fibres \& Textiles in Eastern Europe, 20(1), 57-63 (2012).

5. K. Khairu, I. Raman, M. A. S. Mohamed, M. Ibrahim, S. Wahab, Parameter Optimerization for Photo Polymerization of Microstereolithography, In Advanced Materials Research (Vol. 626, pp. 420-424). Trans Tech Publications (2013).

6. K. Suganuma, Introduction to Printed Electronics, 74, p.130 (2014).

7. K. Crippen, P. Tang, P. Mulready, DuPont Lycra shifts emphasis to global brand management, Journal of Product \& Brand Management, Vol. 4 (1995). 